\title{
Unsteady Thermocapillary Migration of Bubbles
}

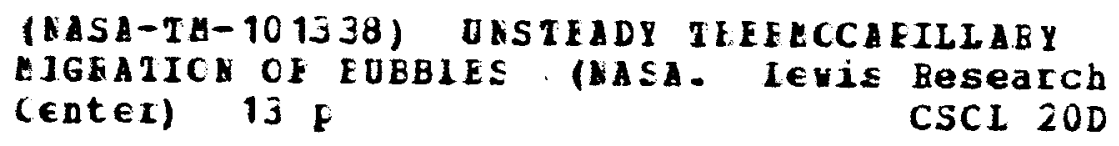

Loren H. Dill

Lewis Research Center

Cleveland, Ohio

and

R. Balasubramaniam

Case Western Reserve University

Cleveland, Ohio

Prepared for the

3rd International Colloquium on Drops and Bubbles sponsored by NASA Jet Propulsion Laboratory

Monterey, California, September 18-21, 1988 


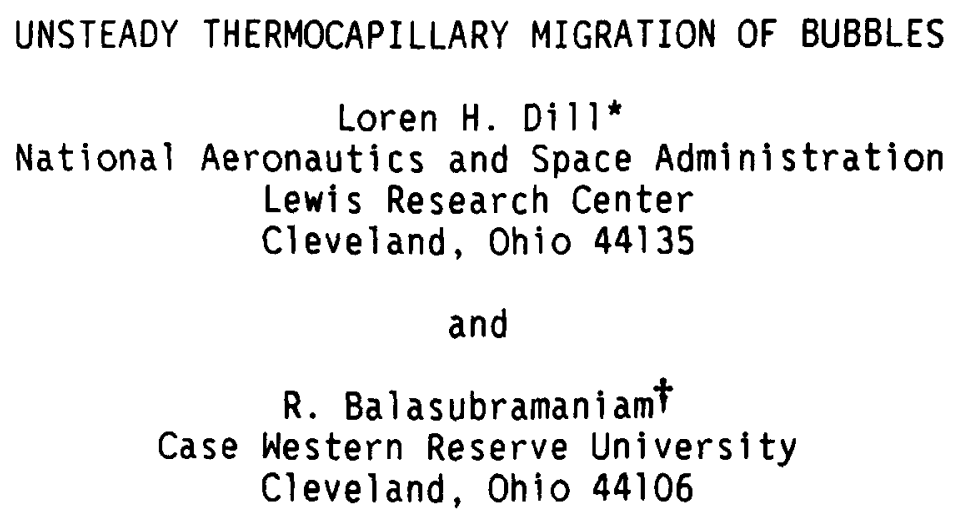

Upon the introduction of a gas bubble into a liquid possessing a uniform thermal gradient, an unsteady thermocapillary flow begins. Ultimately, the bubble attains a constant velocity. This theoretical analys is focuses upon the transient period for a bubble in a microgravity environment and is restricted to situations wherein the flow is sufficiently slow such that inertial terms in the Navier-Stokes equation and convective terms in the energy equation may be safely neglected (i.e., both Reynolds and Marangoni numbers are small). The resulting linear equations were solved analytically in the Laplace domain with the Prandtl number of the liquid as a parameter; inversion was accomplished numerically using a standard IMSL routine. In the asymptotic long-time limit, our theory agrees with the steady-state theory of Young, Goldstein, and Block. The theory predicts that more than 90 percent of the terminal steady velocity is achieved when the smallest dimensionless time, i.e., the one based upon the largest time scale--viscous or thermal--equals unity.

\footnotetext{
*National Research Council - NASA Research Associate.
} †NASA Resident Research Associate. 


\section{INTRODUCTION}

Forces other than gravity are expected to often dominate the migration of bubbles in outer space. In particular, thermocapillary forces will cause bubbles to migrate in a thermal gradient. Consider the bubble depicted in Fig. 1. The interface next to the hot liquid will be hotter than that next to the cold liquid. Assuming that surface tension varies inversely with temperature, as is often the case, the cold interface will have a higher surface tension than the hot interface. The thermocapillary stresses are such that near the interface liquid flows from the hot side to the cold side, as indicated by the arrows. Viscous stresses within the fluid oppose these thermocapillary stresses, causing the bubble to migrate toward the hot side. If the thermal gradient is constant throughout the region, the analys is of Young et al. (1959) revealed that the bubble will ultimately migrate with a steady velocity.

In the processing of materials in outer space, nonisothermal bubble-liquid systems will be common. Control of these systems will require a good understanding of thermocapillary migration. For example, bubbles form in glass melts during the manufacturing process; their removal is essential for the glass to be useful. On Earth, gravitational forces aid in their removal. During the containerless processing of glass in the microgravity environment of space, thermocapillary-forces are expected to assist their removal (Mattox et al. 1982, Subramanian 1981). Thermocapillary migration may al so be important in the design of two-phase heat exchangers for use in outer space. A poor design may unexpectedly permit bubbles to migrate en masse to the heating surface. A layer of gas next to the hot surface would act as a thermal insulator and prevent the efficient transfer of heat to the liquid phase.

In contrast to prior experimental and theoretical studies on thermocapillary migration (Balasubramaniam and Chai 1987, Hardy 1979, Mattox 
et al. 1982, Subramanian 1981, Szymczyk et a1. 1987, Young et a1. 1959), which focus upon steady systems, we here consider the unsteady development of thermocapillary migration. Analysis of unsteady thermocapillary migration is important for several reasons. First, to study the steady state, one must be able to estimate the time required for transients to die out. Also, some unexplained results of supposedly steady-state experiments (Neuhaus and Feuerbacher 1986, Siekmann et al. 1986), e.g., velocities less than predicted steady-state values, may possibly be explained by an unsteady analysis. Lastly, the transient period is expected to be long for relatively large bubbles; a theory for their transport will be useful.

\section{FORMULATION}

Consider a thermocapillary experiment conducted in outer space as shown in Fig. 2. An enclosure consisting of end plates maintained at different temperatures and of insulated side walls is filled with a liquid. In the absence of buoyancy effects, there is no fluid motion and, as depicted in the figure, the temperature field becomes linear when thermal equilibrium is obtained. The experiment then begins with the introduction of a small gas bubble. We neglect the small immediate effect of the bubble's introduction upon the velocity and temperature fields. Soon thereafter the conduction of heat to the interface causes thermocapillary stresses to develop and migration to begin. As is commonly done, we assume that both viscosity and density of the liquid phase are essentially constant throughout the volume, that transport processes within the bubble phase may be neglected relative to those in the liquid phase, and that surface tension is a linear function of interfacial temperature. To be definite, we assume that $\sigma_{T}=d a / d T$ is negative such that migration is toward the hotter fluld. We also assume that the bubble remains spherical during the acceleration; this is subject to a posteriori verification. Finally, we assume that both fluid inertia and the 
convective transport of heat may be neglected. These latter assumptions restrict the analysis to low Reynolds and Marangoni (thermal Peclet) numbers. Given the above assumptions, the dimensionless temperature field $T$ obeys the unsteady energy equation

$$
\operatorname{Pr} \frac{\partial T}{\partial t}=\nabla^{2} T
$$

in the region outside of the bubble. Here, the liquid's Prandtl number $\operatorname{Pr}=v / \alpha$, the ratio of kinematic viscosity to thermal diffusivity, appears multiplying the left-hand side. The variable $t$ is time divided by the viscous time scale $a^{2} / v$, with the characteristic length scale "a" being the radius of the bubble. The dependent variable $T$ is the scaled difference between the actual temperature and that undisturbed temperature which prevails in the plane of the bubble's center. The characteristic temperature scale is the product $a A$, where $A$ is the undisturbed temperature gradient in the system. Eq. (1) is written with the bubble's center as the origin of the coordinate system.

Similarly, the velocity field obeys the unsteady Stokes equation

$$
\frac{\partial v}{\partial t}=-\left(\nabla p+\frac{d U}{d t}\right)+\nabla^{2} v
$$

in a reference frame moving with the bubble. The vector $\mathbf{v}$ is the scaled difference between the fluid's and bubble's velocities. The velocity scale for both $v$ and $U=k U(t)$, the scaled bubble velocity, is the positive quantity $-\sigma_{T} a A / \mu$. Because the bubble's reference frame is noninertial, there appears in Eq. (2) the fictitious force $d U(t) / d t$. This force adds to the gradient of the hydrodynamic pressure $p$ and vanishes when a steady bubble velocity is obtained.

At the interface, viscous and thermocapillary stresses balance at each point. Now both $T$ and $v$ are axisymmetric with respect to an axis that 
passes through the bubble's origin and is parallel to the unit vector $k$, which points in the direction of the temperature gradient. It follows that $T=T(r, \theta, t)$ and $v=v(r, \theta, t)$ and that the stress balance is given by

$$
\left.\frac{\partial}{\partial r}\left(\frac{{ } \theta}{r}\right)\right|_{r=1}=\left.\frac{\partial T}{\partial \theta}\right|_{r=1} .
$$

Here, $r$ is the dimensionless radial coordinate and $\theta$ is the polar angle; these are depicted in Fig. 2. Since the flow is axisymmetric, the vector $v$ has only two nonzero components: $v_{r}$ and $v_{\theta}$, the velocities in the radtal and polar directions, respectively.

Because we neglect bubble inertia and gravitational forces, the total hydrodynamic force $F^{h}$ on the bubble vanishes for all $t>0$ :

$$
F^{h}(t)=\int_{0}^{2 \pi} d \phi \int_{0}^{\pi} n \cdot P(1, \theta, t) \sin \theta d \theta=0
$$

Here, $\boldsymbol{n}$ is a unit vector normal to the spherical bubble surface and $P(r, \theta, t)$ is the hydrodynamic pressure dyadic (tensor).

We briefly mention the remaining conditions on the temperature and velocity fields. The undisturbed temperature and velocity fields prevall (1) Initially for all space outside the bubble and (2) far from the bubble for all time. The neglect of thermal transport within the bubble requires a zero thermal flux normal to the bubble surface for $t>0$. Because the bubble does not change in size, the radial velocity $v_{r}$ vanishes at the interface. Lastly, the assumption of incompressibility requires $\nabla \cdot v=0$.

\section{SOLUTION}

To solve this system of equations, we defined a modified pressure field such that its gradient is given by the quantity within parentheses in Eq. (2), introduced the streamfunction for the axisymmetric flow, and then applied the Laplace transform technique to replace the variable $t$ with the parameter $s$. 
Analytic functions were found for the transformed temperature and streamfunction fields that satisfy the differential equations and all conditions. The solution yielded the following expression for the transformed, dimensionless bubble velocity $O(s)$ :

$$
\bar{U}(s)=\frac{\frac{1}{s}\left(1+\frac{1+\sqrt{s P r}}{2+2 \sqrt{s P r}+s P r}\right)}{\left[3+\frac{s(3+\sqrt{s})}{6(1+\sqrt{s})}\right]}
$$

Multiplication of this expression by $s$ and taking the limit as $s$ approaches zero yields a dimensionless terminal velocity of $1 / 2$, in agreement with the analysis of Young et al. (1959). We also applied the initial-value theorem twice to Eq. (5) to determine $\mathrm{dU} / \mathrm{dt}$ at $t=0$. The first use of the theorem gave the expected result that $U(0)=0$. The second use gave

$$
\left.\frac{d U}{d t}\right|_{t=0}=6 .
$$

The step-function character of the acceleration precluded further application of the initial-value theorem.

To confirm that the bubble remains spherical throughout the acceleration period, we applied our analytic result for the transformed stream function to the transformed normal stress condition. The exact satisfaction of this condition indicates that there is no deviation from the spherical shape.

Numerical inversion via an IMSL routine gave the bubble velocity as functions of time with Prandt 1 number as a parameter. Figure 3 is a log-log plot of the dimensionless velocity versus dimensionless time for Prandtl numbers of $0.01,1.0$, and 100 .

\section{DISCUSSION}

The two most interesting features of Fig. 3 are that (1) all three curves are nearly linear before a transition to the terminal velocity and (2) the 
dimensionless time to the terminal velocity ranges from order one to order 100, depending upon the Prandt l number. These features are discussed below.

For $t \ll 1$, the dimensionless velocity $U(t)$ is given

approximately by

$$
U(t) \sim 6 t
$$

The value of 6 (cf Eq. (6)) derives from the acceleration calculated from application of the initial value theorem. Equation ( 7 ) is represented in Fig. 3 as the dashed line; it fits the data reasonably well for small values of $t$.

Written in dimensional form, Eq. (7) becomes

$$
U^{*}\left(t^{*}\right) \sim 6\left(\frac{-\sigma_{T} A}{\rho a}\right) t^{*}
$$

for $t^{*} v / a^{2} \ll 1$. (The raised asterisks denote dimensional quantities.) According to Eq. (8), the leading term in the short-time expansion for the dimensional velocity is independent of viscosity. This result is analogous to that for a drop or solid sphere accelerating from rest due to buoyancy forces (Chisnell 1987). Though viscosity does not appear explicitly in Eq. (8), it does appear in the time scale and thus affects the values of $t^{*}$ for which the relation is valid. For highly viscous liquids or small bubbles, the relation is valid only briefly. On the other hand, for low-viscosity liquids or large bubbles, Eq. (8) is likely to apply for a much longer period of time.

Another interesting feature of $\mathrm{Eq}$. (8) is that the bubble's acceleration is inversely proportional to its radius. Consider two bubbles of different radii released simultaneously into the same system. During the time regime for which Eq. (8) is applicable for both bubbles, the smaller bubble may be expected to move faster than the larger one, even though the terminal velocity of the smaller bubble, 


$$
U^{*}(\infty)=\frac{-\sigma_{T} \mathrm{aA}}{2 \mu} \text {, }
$$

is less.

Our focus now shifts to estimating the duration of unsteady migration. According to Fig. 3 , the time $t$ to a terminal velocity is of order unity if the $\operatorname{Pr} \leq 1$, but of order 100 if $\operatorname{Pr}=100$. (Recall that the factor used to nondimensionalize time was the viscous scale $a^{2 / \nu}$.) It thus appears that for $\operatorname{Pr} \leq 1$, the viscous scale is appropriate, but for $\operatorname{Pr} \geq 1$, the product of the Prandtl number and the viscous scale is appropriate. This product equals $\mathrm{a}^{2} / \alpha$, which is the characteristic thermal time scale for the system. Observe that for any value of $\mathrm{Pr}$, the larger of the viscous or thermal scales appears to be a good estimate of the transient period.

The above rule to predict the time to steady state was based upon graphical data for three curves. For a more quantitative picture, we examined numeric data for five liquids having Prandtl numbers in the range $0.01 \leq \operatorname{Pr} \leq$ 100. For each Prandtl number, we determined the dimensionless times $\tau_{90}$ and $\tau_{95}$ (based upon the appropriate scale) for a bubble to attain 90 and 95 percent of the terminal velocity. All results (Table 1) are of order unity and thus confirm that the rule is correct.

To understand why the above scaling works, observe that if $\operatorname{Pr} \ll 1$, the thermal field attains its steady-state value while the fluid motion is just beginning to develop. The time for the velocity field to reach its steady state is thus governed by the viscous time scale. If $\operatorname{Pr} \gg 1$, the fluid motion, which is driven by thermocapillary forces and thus by the temperature field, becomes quasistatic with a slowly-developing thermal field. In this case, it is the thermal time scale that determines when the bubble reaches its terminal velocity. 


\section{SUMMARY}

The unsteady thermocapillary migration of a bubble within an otherwise quiescent liquid was studied via analytical methods. Within the same system, small bubbles are predicted to initially move more rapidly than larger ones, even though their terminal velocities are less. The larger of the viscous and thermal time scales is a good estimate of the duration of the unsteady migration period.

\section{REFERENCES}

1. Balasubramaniam, R.; and Chai, An-Ti: Thermocapillary Migration of Droplets: An Exact Solution for Small Marangonl Numbers. J. Colloid Interface Sci., vol. 119, no. 2, Oct. 1987, pp. 531-538.

2. Chisne11, R.F.: The Unsteady Motion of a Drop Moving Vertically Under Gravity. J. Fluid Mech., vol. 176, Mar. 1987, pp. 443-464.

3. Hardy, S.C.: The Motion of Bubbles in a Vertical Temperature Gradient. 3. Colloid Interface Sci., vol. 69, no. 1, Mar. 15, 1979, pp. 157-162.

4. Mattox, D.M., et al.: Thermal-Gradient-Induced Migration of Bubbles in Molten Glass. J. Am. Ceram. Soc., vol. 65, no. 9, Sept. 1982, pp. 437-442.

5. Neuhaus, D.; and Feuerbacher, B.: Bubble Motions Induced by a Temperature Gradient. Proceedings of the 6th European Sympostum on Material Sciences under Microgravity Conditions, ESA SP-256, European Space Agency, Paris, France, 1986, pp. 241-244.

6. Siekmann, J., et al.: Experimental Investigation of Thermocapillary Bubble and Drop Motion Under Microgravity. Proceedings of the 6 th European Symposium on Material Sciences Under Microgravity Conditions, ESA SP-256, European Space Agency, Paris, France, 1986, pp. 179-182. 
7. Subramanian, R.S.: Slow Migration of a Gas Bubble in a Thermal Gradient. AIChE J., vol. 27, no. 4, July 1981, pp. 646-654.

8. Szymczyk, J.A.; Wozniak, G.; and Siekmann, J.: On Marangoni Bubble Motion at Higher Reynolds- and Marangoni-Numbers Under Microgravity. Appl. Microgravity Tech., vol. 1, no. 1, 1987, pp. 27-29.

9. Young, N.O.; Goldstein, J.S.; and Block, M.J.: The Motion of Bubbles in a Vertical Temperature Gradient. J. Fluid Mech., vol. 6, pt. 3, Oct. 1959, pp. 350-356.

TABLE 1. - DIMENSION-
LESS TIMES TO TERMINAL
VELOCITY
\begin{tabular}{|c|r|r|}
\hline$P r=v / \alpha$ & $\tau_{90}$ & \multicolumn{1}{c|}{$\tau_{95}$} \\
\hline 0.01 & 0.28 & 0.47 \\
.10 & .30 & .52 \\
1.0 & .58 & 1.10 \\
10.0 & .29 & .68 \\
100.0 & .26 & .65 \\
\hline
\end{tabular}


IF $\sigma_{T}=\frac{d \sigma}{d T}<0, \ldots$

HOT LIQUID

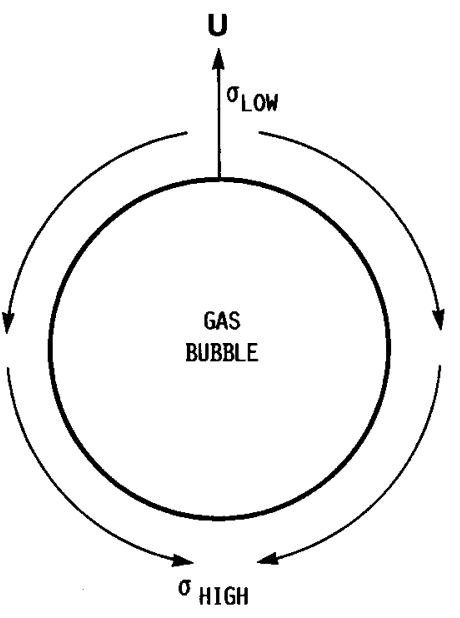

COLD LIQUID

FIGURE 1. - ORIGINS OF THERMOCAPILLARY MIGRATION. VARIATIONS IN SURFACE TENSION ALONG A NONISOTHERMAL INTERFACE CAUSE THERMOCAPILLARY SHEARING STRESSES, WHICH VISCOUS SHEARING STRESSES TRANSMIT TO THE LIQUID. LIQUID IMPOSES A REACTION FORCE UPON THE BUBBLE, CAUSING IT TO SWIM TOWARDS THE HOT LIQUID WITH A VELOCITY U(t).

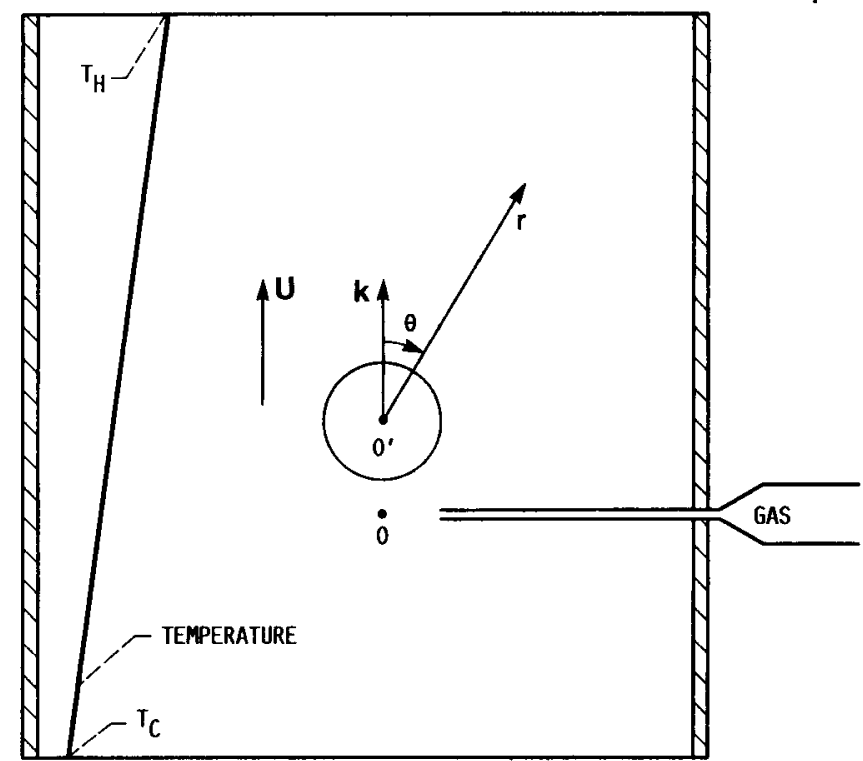

FIGURE 2. - SCHEMATIC OF A THERMOCAPILLARY EXPERIMENTAL CELL IN ZERO GRAVITY. IN TYPICAL EXPERIMENTS. A LINEAR TEMPERATURE FIELD IS ESTABL ISHED PRIOR TO THE INTRODUCTION OF A GAS BUBBLE AT POINT 0. THERMOCAPILLARY ACTION CAUSES THE BUBBLE TO MIGRATE WITH THE UNSTEADY VELOCITY U(1) IN THE DIRECTION OF THE THERMAL GRADIENT. EVENTUALLY. THE MIGRATION VELOCITY BECOMES STEADY.

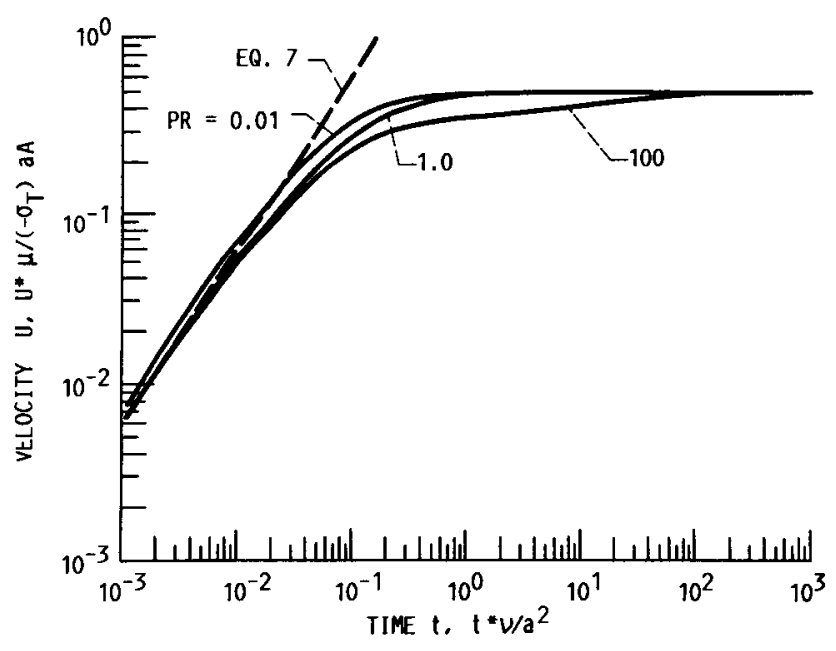

FIGURE 3. - DEVELOPMENT OF THERMOCAPILLARY MIGRATION. EACH SOLID CURVE REPRESENTS A PLOT OF THERMOCAPILLARY MIGRATION VELOCITY $U(t)$ VERSUS TIME $t$ FOR A SPECIFIED PRANDTL NUMBER PR $=v / \alpha$. THE DASHED LINE PROVIDES A GOOD ESTIMATE OF THE VELOCITY FOR SMALL VALUES OF $t$. 


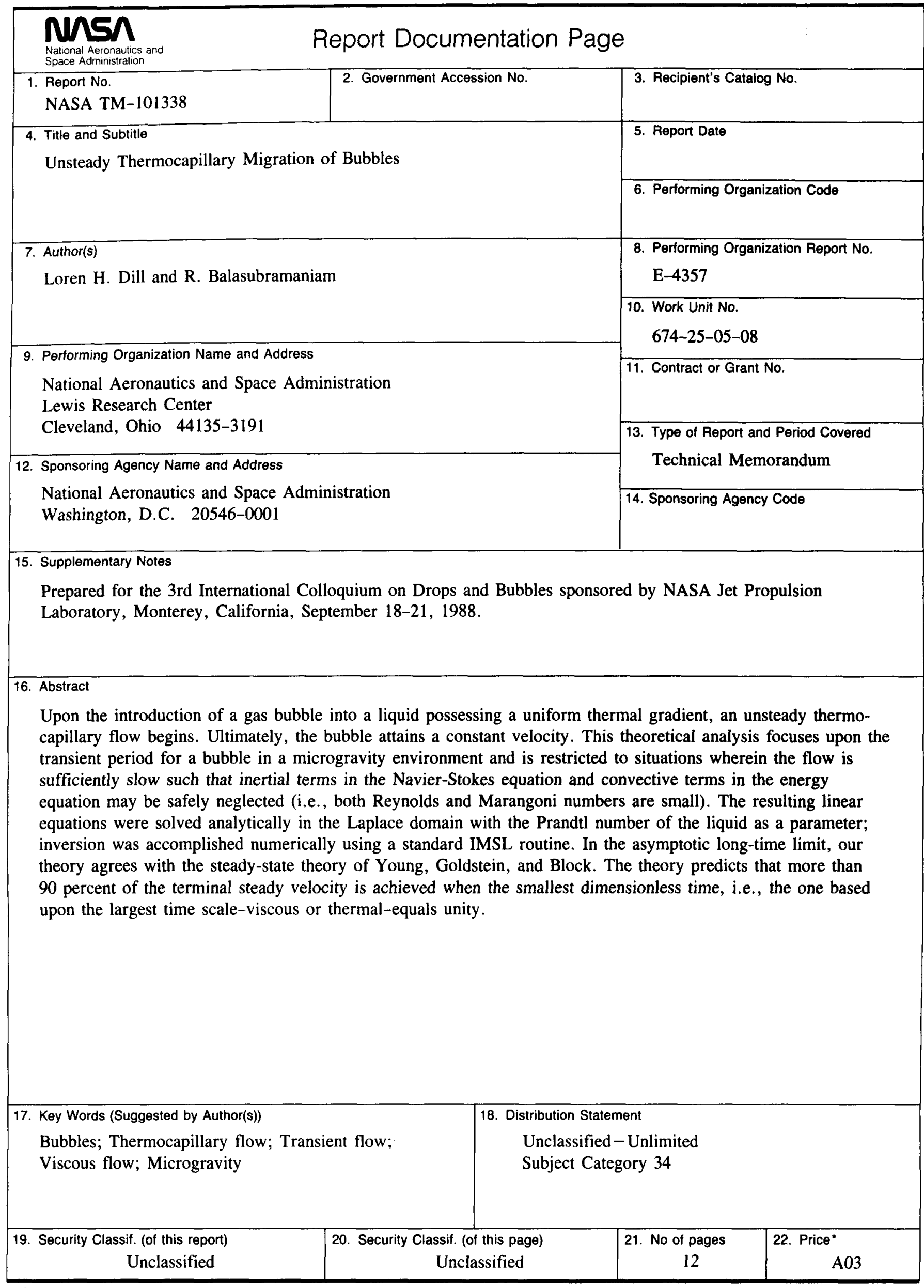

